\title{
A case of suicide by self-injection of adrenaline
}

\author{
Cristian Palmiere $^{1} \cdot$ Fabien Bévalot $^{2} \cdot$ Daniel Malicier $^{3} \cdot$ Eric Grouzmann $^{4}$. \\ Tony Fracasso ${ }^{1} \cdot$ Laurent Fanton $^{3}$
}

Accepted: 16 July 2015/Published online: 2 August 2015

(C) Springer Science+Business Media New York 2015

\begin{abstract}
Adrenaline (epinephrine) auto-injectors provide life-saving pre-hospital treatment for individuals experiencing anaphylaxis in a community setting. Errors in handling adrenaline auto-injectors, particularly by children and healthcare professionals, have been reported. Reports of adrenaline overdoses are limited in the medical literature. In most of these cases, accidental adrenaline administration results from medical error. Exogenous administration of catecholamine is responsible for cardiovascular and metabolic responses, which may cause supraventricular tachycardia, ventricular dysrhythmias and myocardial ischemia. The authors present a unique autopsy case involving a 34 year-old woman who intentionally self-injected adrenaline using an adrenaline auto-injector as part of a suicide plan. Catecholamines and metanephrines were measured in peripheral and cardiac blood as well as urine and vitreous humor. Based on the results of all postmortem investigations, the cause of death was determined to be cardiac dysrhythmia and cardiac arrest following adrenaline selfinjection.
\end{abstract}

Keywords Adrenaline · Auto-injector · Suicide · Postmortem biochemistry

Cristian Palmiere

cristian.palmiere@chuv.ch

1 CURML, Centre Hospitalier Universitaire Vaudois (CHUV), Lausanne, Switzerland

2 Laboratoire LAT LUMTOX, Lyon, France

3 Faculté de Médecine, Institut médico-légal, Lyon, France

4 Service of Biomedicine, Catecholamine and Peptides Laboratory, Centre Hospitalier Universitaire Vaudois (CHUV), Lausanne, Switzerland

\section{Introduction}

Anaphylaxis is an acute, serious, generalized or systemic, allergic or hypersensitivity reaction, with varied mechanisms and clinical presentations, that can be life-threatening or fatal. The reaction is rapid in onset and results from the sudden, systemic release of mediators from mast cells and basophils [1-3]. Triggers may include food, stinging insect venoms, drugs, biological agents, radiocontrast media, latex or unknown causes [1]. Adrenaline (epinephrine) delivered intramuscularly into the anterolateral aspect of the mid-thigh through prefilled auto-injectors (adrenaline auto-injection device, AAD) is recommended as the initial, potentially life-saving treatment of choice. This should be used as emergency supportive therapy for anaphylaxis in the outpatient setting while medical care is sought $[1,2,4-7]$.

Case reports describing unintentional injection with $\mathrm{AAD}$ have been published. Errors in handling adrenaline auto-injectors, particularly by children and healthcare professionals, can result in severe consequences. Here we present a unique autopsy case involving a 34 year-old woman who intentionally self-injected adrenaline using an $\mathrm{AAD}$ as part of a suicide plan.

\section{Case history}

The body of a 34 year-old Caucasian woman was found outside her home, lying in the grass, extended in a supine position, fully clothed and partially covered. The deceased's clothes were intact and did not reveal any evidence of struggle or violence. No blood or vomit was present on or around the body. Rigor mortis was present in the major and minor joints. Reddish purple postmortem lividity was 
present on the back and dependent body parts in the supine position. Lividity matched the position of the body and could be partially eliminated by finger pressure. Two adrenaline auto-injection devices (Anapen Auto-Injector $0.15 \mathrm{mg}$ of adrenaline in $0.3 \mathrm{ml}$ solution) were found in the immediate vicinity of the body. No cardiopulmonary resuscitation attempts were performed. The door of the house was unlocked. No tools or weapons were noted inside the house. A suicide note was discovered in the living room in which the woman purportedly mentioned that she was ending her life. According to medical records obtained from local health services and general practitioners, the woman had suffered from generalized anxiety disorder and severe depression since her husband's suicide 7 months previously. Her present medical treatment consisted of mirtazapine, citalopram, prazepam and ciamemazine (ciamepromazine), although she was described as a non-compliant patient. One of her children was allergic to bee and wasp sting venom, and for this reason she had adrenaline auto-injection devices available at home.

The macroscopic external examination of the body at the death scene essentially revealed two skin injection marks at the left thigh (the woman wore a skirt with no pantyhose) and multiple ant bite marks on the left ear and upper legs. No bleeding wounds were observed at any site. Due to the unclear circumstances of death and the potential involvement of a third party, a medico-legal autopsy was ordered by the public prosecutor.

The deceased was $170 \mathrm{~cm}$ tall and weighed $54 \mathrm{~kg}$. External examination performed at the medico-legal-center was unremarkable except for the presence of two fresh injection marks on the left thigh and the presence of multiple, dry ant bites elsewhere. The examination did not show evidence of other external traumas.

At autopsy, no skull, rib or sternum fractures were observed. The heart weighed $260 \mathrm{~g}$ and revealed a degree of cavity dilatation. The lungs were relatively edematous and congested (left, $420 \mathrm{~g}$; right, $535 \mathrm{~g}$ ). The liver weighed $1225 \mathrm{~g}$ and was relatively congested. The esophagus and the stomach contained an estimated $180 \mathrm{~g}$ of brownish liquid material. No identifiable pill components or fragments were found in the stomach contents or upper gastrointestinal tract. The urinary bladder contained $220 \mathrm{ml}$ of yellow urine. The brain weighed $1330 \mathrm{~g}$ and displayed edema with slight flattening of the gyri and mild cerebellar tonsillar grooving. No other abnormalities were observed elsewhere in the body.

Microscopically [hematoxylin-eosin (HE) stain], the heart revealed no evidence of subendocardial hemorrhage or acute myocardial ischemia. Contraction band necrosis was not observed. The lungs showed congestion and edema as well as areas of emphysema. Acute generalized visceral congestion was also noted. Neuropathology was unremarkable. Histology (HE) was also performed on tissues (epidermis, derma, and hypodermic adipose tissue) sampled from the injection sites on the left thigh, revealing fresh, focal hemorrhagic areas.

Peripheral blood from the femoral vein, cardiac blood, bile, urine, gastric content, pericardial fluid and vitreous humor were recovered for toxicological and biochemical analyses and immediately frozen at $-20{ }^{\circ} \mathrm{C}$ until analysis.

Toxicology included blood ethanol and other volatile compound determination as well as general screening for common drugs and illegal substances by gas chromatography-mass spectrometry (GC-MS) using commercial mass spectrum libraries, high-performance liquid chromatography with diode-array detection (HPLC-DAD) and headspace-gas chromatography flame ionization detection (HS-GC-FID). Toxicological analysis failed to detect ethanol and revealed the presence of mirtazapine, citalopram, prazepam and ciamemazine in peripheral blood within therapeutic ranges.

Catecholamines (adrenaline and noradrenaline), metanephrines (metanephrine, normetanephrine) and 3-methoxytyramine were analyzed in vitreous humor, urine, peripheral and cardiac blood using HPLC with amperometric detection, as previously described [8]. The clinical reference intervals concerning free and total metanephrines in plasma, fractionated metanephrines in urine and urinary catecholamines were obtained from the clinical literature and were used to interpret the postmortem results [9, 10]. Results obtained from vitreous analysis were interpreted using the clinical plasma reference values.

Results in vitreous humor, cardiac, and peripheral blood were expressed in $\mathrm{nmol} / \mathrm{l}$. Results in urine were expressed in $\mathrm{nmol} / \mathrm{mmol}$ creatinine (Table 1 ).

The adrenaline concentration in cardiac blood $(79.7 \mathrm{nmol} / \mathrm{l})$ was markedly increased compared to the concentration found in peripheral blood $(0.33 \mathrm{nmol} / \mathrm{l})$. It was also significantly higher than the concentrations usually measured in our facility in cases of cardiac deaths (mean value in cardiac blood $30 \mathrm{nmol} / \mathrm{l}$, according to the results obtained in our facility in 100 medico-legal cases).

Analogously, metanephrine and normetanephrine levels in cardiac blood (30.02 and $19.37 \mathrm{nmol} / \mathrm{l}$, respectively) were markedly increased compared to the concentrations found in peripheral blood ( 8.15 and $10.36 \mathrm{nmol} / \mathrm{l}$, respectively), though the latter were higher than plasma reference values.

In addition, metanephrine and normetanephrine levels in the cardiac blood were significantly higher than those normally measured in our facility in cases of cardiac deaths (mean metanephrine value in cardiac blood $21 \mathrm{nmol} / \mathrm{l}$, mean normetanephrine value $11 \mathrm{nmol} / \mathrm{l})$. 
Table 1 Summary of catecholamine and metanephrine determination in peripheral blood, cardiac blood, urine, and vitreous humor

\begin{tabular}{llllll}
\hline & Adrenaline & Noradrenaline & Metanephrine & Normetanephrine & $3-$ methoxytyramine \\
\hline Peripheral blood (nmol/l) & $0.33[0.02-1.23]$ & $1.45[0.64-6.55]$ & $8.15[0.03-0.85]$ & $10.36[0.04-1.39]$ & $0.38[<0.06]$ \\
Cardiac blood (nmol/l) & $79.7[0.02-1.23]$ & $8.78[0.64-6.55]$ & $30.02[0.03-0.85]$ & $19.37[0.04-1.39]$ & $13.95[<0.06]$ \\
Urine (nmol/mmol) creatinine & $101[<22]$ & $489[<45]$ & $337[<200]$ & $872[<250]$ & $371[<150]$ \\
Vitreous humor (nmol/l) & $0.10[0.02-1.23]$ & $0.78[0.64-6.55]$ & $0.15[0.03-0.85]$ & $0.12[0.04-1.39]$ & $0.01[<0.06]$ \\
\hline
\end{tabular}

Plasma and urine reference values $[9,10]$ are indicated in brackets. Results obtained from vitreous analysis were interpreted using the clinical plasma reference values

Catecholamines and metanephrines in vitreous were lower than or within plasma clinical reference values.

Lastly, urine analysis revealed extremely high levels of all tested compounds (catecholamines, metanephrines, and 3-methoxytyramine) compared to urinary clinical reference values as well as to the concentrations normally measured in our facility in hypothermia fatalities and cardiac deaths (Table 1).

Based on the results of all investigations, the cause of death was determined to be cardiac dysrhythmia and cardiac arrest following adrenaline self-injection. Direct third party involvement was excluded and the manner of death was listed as suicide. The case was not pursued any further by the public prosecutor.

\section{Discussion}

Catecholamines are a class of adrenergic compounds, chemically characterized by two adjacent hydroxyl groups on a benzene ring. These can either be administered exogenously or secreted endogenously in response to environmental stress [11-13].

Human plasma contains six readily detectable catechols, the three catecholamines (adrenaline, noradrenaline, and dopamine), their precursor levodopa (L-DOPA), and their deaminated metabolites. Endogenous catecholamines undergo a complex fate mediated by several enzymes in various combinations. Since these enzymes are expressed differently in various tissues, circulating product levels have distinctive sources and reflect specific aspects of sympathetic neuronal and adrenomedullary hormonal system functions. The enzyme catechol- $O$-methyltransferase catalyzes the $O$-methylation of the 3 -hydroxyl group of most catechols. 3-methoxytyrosine is the $O$-methylated derivative of L-DOPA, 3-methoxytyramine is mainly that of dopamine, normetanephrine that of noradrenaline and metanephrine that of adrenaline. The corresponding 3-Omethylated metabolite of dopamine (3-methoxytyramine) is not generally considered a metanephrine, a term that refers to metanephrine and normetanephrine [12, 13].
In healthy individuals, endogenous catecholamines are usually released in sufficient amounts to respond to stressful situations in a self-regulating manner. Conversely, due to their activity on the cardiovascular system, exogenous catecholamine administration requires careful titration to achieve the desired effects. Catecholamines are indeed capable of altering blood pressure, pulse, electrolyte homeostasis, and blood flow to most organ systems under neuro-chemical control [11].

Adrenaline acts on myocardium $\beta 1$-receptors to create positive inotropic and chronotropic action, which lead to increased contractility and heart rate, shorter and more powerful systole, increased cardiac output, and significantly increased oxygen consumption. Stimulation of both $\alpha$ and $\beta$ adrenergic receptors is responsible for massive vasoconstriction. The sudden increase in adrenergic tone and vasoconstriction in the capillary resistance vessels of the kidney may cause significant decreases in renal blood flow and acute failure [14].

Adrenaline-induced hypokalemia results from $\beta 2$ adrenoreceptor stimulation mediated by cyclic adenosine monophosphate, which activates membrane sodium and potassium adenosine triphosphatase $\left(\mathrm{Na}^{+}-\mathrm{K}^{+}\right.$ATPase), causing potassium influx from extracellular space to intracellular space. Increasing the number of enzyme molecules in the cell membrane or accelerating the rate of activity of existing enzyme molecules may enhance $\mathrm{Na}^{+}-$ $\mathrm{K}^{+}$ATPase activity. Therefore, potassium stored in extracellular space shifts into intracellular space. Insulin, renin, or aldosterone, do not mediate adrenaline-induced hypokalemia [15].

Unintentional AAD injections have been infrequently reported in the medical literature in the past. Though adverse outcomes were not reported in a consistent manner, serious injury and permanent sequelae were described as rare. Most unintentional adrenaline administration from auto-injectors are due to poor knowledge of proper AAD use among patients, families and health care professionals, though device malfunctions and deficiency in device design may also be occasionally implicated [16, 17]. 
Anapen Auto-Injectors are automatic injectors designed to give a single fixed dose of adrenaline. The administration of Anapen requires the removal of a black needle shield and a gray safety cap. The device must be held against the outer mid-thigh and is activated by depressing a red button with the thumb. It should be held in place for ten seconds after activation and can be injected through a single layer of clothing. The device is provided with stepby-step instructions.

Reports of adrenaline overdoses and their outcomes are limited in the medical literature. Accidental adrenaline administration results from medical error in most cases, generally followed by the rapid onset of agitation, tachycardia, hypertension, and dysrhythmias [12, 18-22]. Karch [18] described a case of accidental, intravenous administration of $2 \mathrm{mg}$ adrenaline in a 27-year-old man who developed transient ischemia. Serum catecholamines, drawn approximately $20 \mathrm{~min}$ after adrenaline administration, were ten times higher than normal (dopamine $173 \mathrm{ng} /$ 1, adrenaline $1628 \mathrm{ng} / \mathrm{l}$, and noradrenaline $1972 \mathrm{ng} / \mathrm{l}$ ). Woodard and Brent [12] reported a case of overdose following the recreational injection of an unknown amount of adrenaline (amount estimated between 82.5 and $124 \mathrm{mg}$, more than ten times the dose normally administered), which resulted in acute myocardial infarction and acute renal failure. The patient had extracted adrenaline from an over the counter adrenaline-metered dose inhaler after heating it in the microwave, and added tap water as a diluent. The total volume of fluid injected, including diluents, was thought to be $40 \mathrm{ml}$.

Adrenaline overdoses in newborns and children have been associated with rhabdomyolysis, supraventricular tachycardia, ventricular dysrhythmias, and myocardial ischemia $[13,20]$.

Arfi et al. [19] reported the case of a young patient who inadvertently received adrenaline intravenously rather than subcutaneously. The patient developed myocardial ischemia, transiently raised cardiac enzymes, reduced left ventricular systolic function, pulmonary edema, and pulmonary hemorrhage.

Davidsen et al. [21] described a case of acute myocardial infarction following an accidental, iatrogenic adrenaline injection $(1 \mathrm{mg})$ in a 55-year-old-man. Immediately after receiving adrenaline, the patient presented angina pectoris, dyspnea, and ST-segment elevation. Cardiac enzymes were raised. A coronary angiography revealed $66 \%$ stenosis of the right coronary artery. This is likely to have become critical due to coronary artery spasm related to the adrenaline injection.

In the realm of forensic pathology, catecholamine levels have been investigated in relation to the cause of death by several authors [23-29].
Adrenaline and noradrenaline were shown to be stable in the cadaveric urine, with no significant changes in the concentrations in relation to the postmortem period. On the other hand, catecholamine stability in postmortem blood samples remains an issue of controversy, since they may diffuse from sympathetic nerve ending and adrenal glands into the blood [30].

Zhu et al. [23] measured catecholamine levels in postmortem serum obtained from subclavian and external iliac vein blood as well as left and right heart blood. They observed significantly higher catecholamine concentrations in postmortem serum obtained from any sampling site compared to clinical reference intervals, though levels were usually higher in postmortem serum obtained from right heart blood than in postmortem serum collected elsewhere. Wilke [24] studied catecholamine concentrations in femoral blood, heart blood, cerebrospinal fluid, urine, and vitreous. Contrary to Zhu et al. [23], Wilke [24] observed that absolute values for adrenaline and noradrenaline in heart and femoral blood displayed no significant differences in relation to the cause of death.

In a series of succeeding studies, Ishikawa et al. [25-27] investigated catecholamine levels in postmortem serum from right heart blood, urine, pericardial, and cerebrospinal fluids. These authors observed that catecholamine concentrations in postmortem serum from right heart blood were usually higher than levels in urine (and higher than clinical reference values). Conversely, urinary adrenaline and noradrenaline values fell within clinical serum reference ranges.

It must be emphasized that none of the aforementioned studies pertaining to catecholamine concentrations in biological fluids collected at autopsy mentioned cases of death following voluntary or accidental adrenaline administration. It is therefore difficult to compare the results of the aforementioned reports to those observed in the cases herein described.

On the other hand, only one study to date has evaluated both catecholamine and metanephrine concentrations in urine in medico-legal cases, and no report exists pertaining to metanephrine levels in postmortem serum and vitreous humor [28]. The unavailability of reliable postmortem reference values pertaining to catecholamines and metanephrines in postmortem serum, vitreous humor, and urine is a limitation of this study.

In the living, plasma metanephrines are considered to be more stable than catecholamines [31]. Hence, postmortem serum and/or urine metanephrines could be more suitable biochemical markers of exogenous catecholamine administration, should this hypothesis need to be investigated in the forensic setting. It must be highlighted, however, that the unavailability of forensic studies on these topics 
renders any comparison of our results to other clinical or forensic investigations hazardous.

To the best of our knowledge, no case of suicide following adrenaline self-administration using a prefilled auto-injector has been reported in the forensic literature to date. Within the previously mentioned limits, the circumstantial elements and data obtained from autopsy, histology, toxicology, and biochemistry were not in contradiction with the hypothesis of death due to cardiac dysrhythmia and cardiac arrest following adrenaline selfinjection. Toxicological analysis revealed blood mirtazapine, citalopram, prazepam, and ciamemazine within therapeutic ranges and allowed drug intoxication as the cause of death to be ruled out.

\section{Key Points}

1. Unintentional adrenaline administration from prefilled auto-injectors has been occasionally described in the past. Reports of adrenaline overdoses and their outcome are limited in the medical literature. To date, no case of suicide using adrenaline auto-injection devices has been reported.

2. Exogenous administration of catecholamine is responsible for cardiovascular (increases in heart rate and blood pressure) and metabolic (hypokalemia) responses, which may in turn cause supraventricular tachycardia, ventricular dysrhythmias, and myocardial ischemia.

3. The increased adrenaline and metanephrine concentrations in cardiac postmortem serum and urine in the case herein described might be consistent with the hypothesis of death following adrenaline self-injection.

4. The correlation of circumstantial elements, autopsy observations, histology findings, toxicological data, and postmortem biochemical results are decisive in identifying the cause of death, excluding other possible causes and characterizing the pathophysiological mechanisms involved in the death process.

\section{Compliance with ethical standards}

Conflict of interest The authors have no conflict of interest to declare.

\section{References}

1. Simons FE, Ardusso LR, Bilò MB, Cardona V, Ebisawa M, ElGamal YM, et al. International consensus on (ICON) anaphylaxis. World Allergy Organ J. 2014;7:9.

2. Salter SM, Loh R, Sanfilippo FM, Clifford RM. Demonstration of epinephrine autoinjectors (EpiPen and Anapen) by pharmacists in a randomised, simulated patient assessment: acceptable, but room for improvement. Allergy Asthma Clin Immunol. 2014;10:49.

3. Palmiere C, Comment L, Mangin P. Allergic reactions following contrast material administration: nomenclature, classification and mechanisms. Int J Legal Med. 2014;128:95-103.

4. Quercia O, Emiliani F, Stefanini B, Stefanini GF. The importance of educating subjects entitled to use an adrenaline auto-injector for self-administration. Eur Ann Allergy Clin Immunol. 2014;46: 237-8.

5. Sheikh A, Simons FE, Barbour V, Worth A. Adrenaline autoinjectors for the treatment of anaphylaxis with and without cardiovascular collapse in the community. Cochrane Database Syst Rev. 2012;8:CD008935.

6. Song TT, Worm M, Lieberman P. Anaphylaxis treatment: current barriers to adrenaline auto-injector use. Allergy. 2014;69:983-91.

7. Puglisi G. Epinephrine autoinjectors: importance of the BX rating. Ann Allergy Asthma Immunol. 2014;113:494-5.

8. Grouzmann E, Fathi M, Gillet M, de Torrenté A, Cavadas C, Brunner $\mathrm{H}$, et al. Disappearance rate of catecholamines, total metanephrines, and neuropeptide $\mathrm{Y}$ from the plasma of patients after resection of pheochromocytoma. Clin Chem. 2001;47: $1075-82$.

9. Grouzmann E, Drouard-Troalen L, Baudin E, Plouin PF, Muller B, Grand D, et al. Diagnostic accuracy of free and total metanephrines in plasma and fractionated metanephrines in urine of patients with pheochromocytoma. Eur J Endocrinol. 2010;162: 951-60.

10. Pussard E, Neveux M, Guigueno N. Reference intervals for urinary catecholamines and metabolites from birth to adulthood. Clin Biochem. 2009;42:536-9.

11. Campbell RL. Cardiovascular effects of epinephrine overdose: case report. Anesth Prog. 1977;24:190-3.

12. Woodard ML, Brent LD. Acute renal failure, anterior myocardial infarction, and atrial fibrillation complicating epinephrine abuse. Pharmacotherapy. 1988;18:656-8.

13. Fang W, Chen JY, Fang Y, Huang JL. Epinephrine overdoseassociated hypokylemia and rhabdomyolysis in a newborn. Pharmacotherapy. 2005;25:1266-70.

14. Oeltmann T, Carson R, Shannon JR, Ketch T, Robertson D. Assessment of $O$-methylated catecholamine levels in plasma and urine for diagnosis of autonomic disorders. Auton Neurosci. 2004;116:1-10.

15. Goldstein DS, Eisenhofer G, Kopin IJ. Sources and significance of plasma levels of catechols and their metabolites in humans. J Pharmacol Exp Ther. 2003;305:800-11.

16. Simons FE, Edwards ES, Read EJ Jr, Clark S, Liebelt EL. Voluntarily reported unintentional injections from epinephrine autoinjectors. J Allergy Clin Immunol. 2010;125:419-23.

17. Simons FE, Lieberman PL, Read EJ Jr, Edwards ES. Hazard of unintentional injection of epinephrine from autoinjectors: a systematic review. Ann Allergy Asthma Immunol. 2009;102:282-7.

18. Karch SB. Coronary artery spasm induced by intravenous epinephrine overdose. Am J Emerg Med. 1989;7:485-8.

19. Arfi AM, Kouatli A, Al-Ata J, Arif H, Syed S. Acute myocardial ischemia following accidental intravenous administration of epinephrine in high concentration. Indian Heart J. 2005;57: $261-4$.

20. Davis CO, Wax PM. Prehospital epinephrine overdose in a child resulting in ventricular dysrhythmias and myocardial ischemia. Pediatr Emerg Care. 1999;15:116-8.

21. Davidsen JR, Lambrechtsen J, Egstrup K. ST elevation myocardial infarction after therapeutic injection of adrenaline. Ugeskr Laeger. 2006;168:1137-8.

22. Senthilkumaran S, Menezes RG, Ibrahim SM, Thirumalaikolundusubramanian P. Cardiac anaphylaxis: searching for clarity. Am J Emerg Med. 2014;32:86. 
23. Zhu BL, Ishikawa T, Michiue T, Li DR, Zhao D, Quan L, et al. Postmortem serum catecholamine levels in relation to the cause of death. Forensic Sci Int. 2007;173:122-9.

24. Wilke N, Janssen H, Fahrenhorst C, Hecker H, Manns MP, Brabant EG, et al. Postmortem determination of concentrations of stress hormones in various body fluids-is there a dependency between adrenaline/noradrenaline quotient, cause of death and agony time? Int J Legal Med. 2007;121:385-94.

25. Ishikawa T, Quan L, Michiue T, Kawamoto O, Wang Q, Chen $\mathrm{JH}$, et al. Postmortem catecholamine levels in pericardial and cerebrospinal fluids with regard to the cause of death in medicolegal autopsy. Forensic Sci Int. 2013;228:52-60.

26. Ishikawa $\mathrm{T}$, Inamori-Kawamoto $\mathrm{O}$, Quan L, Michiue T, Chen JH, Wang Q, et al. Postmortem urinary catecholamine levels with regard to the cause of death. Leg Med (Tokyo). 2014;16:344-9.

27. Ishikawa T, Yoshida C, Michiue T, Perdekamp MG, Pollak S, Maeda H. Immunohistochemistry of catecholamine in the hypothalamic-pituitary-adrenal system with special regard to fatal hypothermia and hyperthermia. Leg Med (Tokyo). 2010;12:121-7.

28. Palmiere C, Teresiński G, Hejna P, Mangin P, Grouzmann E. Diagnostic performance of urinary metanephrines for the postmortem diagnosis of hypothermia. Forensic Sci Med Pathol. 2014;10:518-25.

29. Tormey WP, Carney M, FitzGerald RJ. Catecholamines in urine after death. Forensic Sci Int. 1999;103:67-71.

30. Pakanen L, Kortelainen ML, Särkioja T, Porvari K. Increased adrenaline to noradrenaline ratio is a superior indicator of antemortem hypothermia compared with separate catecholamine concentrations. J Forensic Sci. 2011;56:1213-8.

31. Lagerstedt SA, O'Kane DJ, Singh RJ. Measurement of plasma free metanephrine and normetanephrine by liquid chromatography-tandem mass spectrometry for diagnosis of pheochromocytoma. Clin Chem. 2004;50:603-11. 